\title{
Development of a new multi-purpose wrench based on a lathe
}

\author{
Peng Wang ${ }^{1}$, Yang Wang ${ }^{1}$, Haotong $\mathrm{Yu}^{1}$, Na Cheng ${ }^{1}$, Honghua $\mathrm{Hao}^{2}$ and Zhou Yan ${ }^{3, *}$ \\ ${ }^{1}$ School of Mechatronics Engineering, Shenyang Aerospace University, Shenyang, China \\ ${ }^{2}$ School of Aeronautics and Astronautics, Shenyang Aerospace University, Shenyang, China \\ ${ }^{3}$ Engineering Training Center, Shenyang Aerospace University, Shenyang, China
}

\begin{abstract}
In lathe operation, the most frequently used wrenches are chuck wrenches and tool holder wrenches, which are different in structure and function and cannot be replaced, and forgetting to remove the wrenches will cause safety accidents. To solve the above problems, this paper designs a new type of wrench, which integrates the functions of chuck wrench, tool holder wrench and various screwdrivers, and can easily change the form of wrench by certain operation, which improves the production efficiency and eliminates the safety hazards.
\end{abstract}

\section{Introduction}

In the machining workshop, the common wrenches for lathes are chuck wrenches and tool holder wrenches. If the operator forgets to remove them from the chuck or tool holder, the wrenches will easily fly out when starting the lathe, which will endanger the personal safety of the operator and cause damage to the turning tool and guide rail, thus causing safety accidents.

The chuck wrench and the tool holder wrench are each used in use and cannot be replaced, and it is necessary to change these two wrenches frequently, which is easy to take mixed; the two pairs of wrenches take up space and other resources when kept and stored.

In view of the above situation, it is necessary to design a kind of safety multi-wrench, which can combine the functions of chuck wrench, tool holder wrench and many kinds of screwdrivers in one, and the volume is only the size of one wrench, so that there will be no situation of taking the wrong wrench, and it can also reduce the equipment manufacturing cost and save space and resources.

On the other hand, in view of the most important shortcoming and deficiency of the existing chuck or tool holder wrenches, which cause a large number of accidents due to failure to remove them in time, a wrench that can automatically eject the chuck or tool holder is designed so that no accident occurs because the operator forgets to remove the wrench.

\section{Main principle and structural design of safety chuck wrench}

\subsection{Main design principles and program descriptions}

Forhow to realize the key problem ofautomatic ejection of the wrench from the chuck or the tool holder, our design solution is to make a structural design on the wrench body and install a kind of automatic ejection device, so that the wrench can automatically eject from the chuck or the tool holder when the operator no longer applies force to the wrench.

In this scheme, the spring force of the spring is used to realize the automatic ejection function of the wrench, and the reset function of the spring is also used to control and adjust the position and stroke of the work by the spring. Considering that the actual structure of this design is mainly cylindrical, and that cylindrical springs are the most widely used, standardized in manufacture and relatively simple in structure, it was decided to use cylindrical compression springs.

In order to realize the combination of two wrenches into one, the "outer sleeve inner core" type was adopted, and the chuck wrench was placed inside the sleeve of the tool holder wrench.

In addition, a screwdriver head slot is milled out at one end of the lever, so that different types of screwdriver heads can be replaced and installed; various types of replaceable screwdriver heads, such as one screwdriver head, Phillips screwdriver head, square screwdriver head, etc., are installed inside the lever to facilitate the replacement of screwdriver heads.

\footnotetext{
* Corresponding author: sdyanzhou16888@edu.sau.cn
} 


\subsection{Overall structure and important structural design}

\subsubsection{Overall structure and working principle.}

Figure 1 shows a schematic diagram of the structure of the chuck wrench body, taking the existing chuck wrench as a prototype, and the structure is designed as a step shaft.

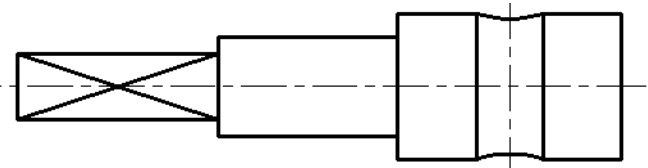

Figure 1. Schematic diagram of chuck trigger body structure.

The overall structure of the new multi-purpose wrench for lathe is shown in Figure 2, including the top of the wrench body, upper spring, slot, force lever, chuck wrench body, lower spring and tool holder wrench sleeve, using the "outer jacket inner core" type, combining the chuck wrench body and tool holder wrench sleeve together.

The chuck wrench body 5 and the tool holder wrench sleeve 7 are the main functional structures, the chuck wrench body 5 is installed inside the tool holder wrench sleeve 7 , and the chuck wrench can extend out of the tool holder wrench sleeve 7 through the lower edge of the inner hole of the tool holder wrench sleeve 7 in the process of downward pressure, and the function of the chuck wrench and the tool holder wrench can be realized through the difference of the limit position; the tool holder wrench sleeve 7 plays the role of protecting the whole wrench body and the spring.

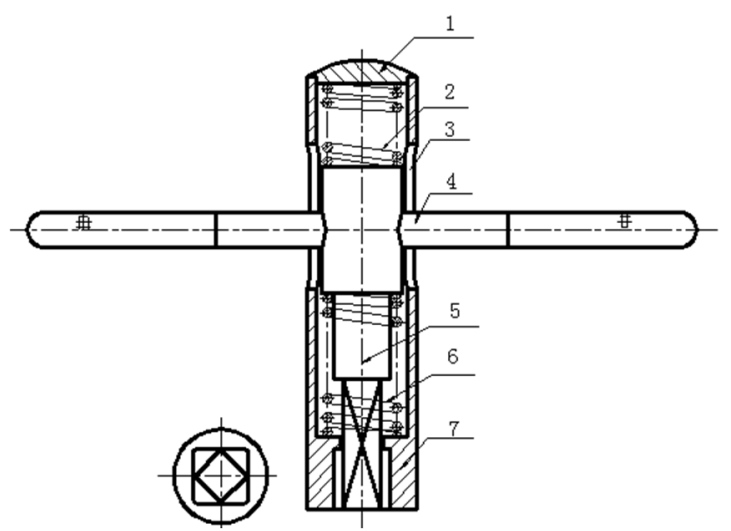

1.top of wrench body 2 . upper spring 3 . slot 4 . application lever

5. chuck wrench body 6 . lower spring 7 . tool holder wrench sleeve

Figure 2. Overall assembly diagram.

The upper spring 2 and lower spring 6 are each installed at the upper and lower shoulders of the chuck wrench body 5 , which can be telescoped during the downward and backward presses of the chuck wrench body 5, respectively, to realize the automatic reset of the wrench body.

The force lever 4 transversely passes through the slot 3 on the sleeve of the knife holder wrench and the chuck trigger body 5 and is fixed on the chuck trigger body 5 , which can slide along the slot 3 and its downward and backward pressing position is restricted by the slot 3 . The inner hollow of the force lever 4 can store the screwdriver bit, Phillips screwdriver bit, square screwdriver bit, etc.

The working principle is as follows: When the chuck tightens or loosens the working piece press hard on the force-applied lever 4 , the lower spring 6 contracts under pressure and the chuck trigger body 5 extends, rotate the force-applied lever 4 hard to tighten or loosen it, after which the force pressed on the force-applied lever 4 is canceled and the force-applied spring 6 is reset, thus pushing the chuck trigger body 5 upward and thus making the chuck trigger body 5 eject from the chuck square hole. When it is necessary to change the tool, let the bottom align with the screw on the tool holder, press hard on the top 1 of the trigger body, the upper spring 2 is contracted by the pressure, rotate the force-applying lever by force thus tightening or loosening the screw, after which the force pressed on the force-applying lever 4 is canceled and the pressured upper spring 2 is reset so that the trigger body is ejected from the screw.

When you need to use a screwdriver, remove the force lever and select the corresponding screwdriver head.

\subsubsection{Structural design of the force bar.}

By designing the inner hexagonal hole at one end of the force application rod, it makes it possible to change the required screwdriver head at any time; the other end is designed with a round hole to place different types of screwdriver heads. This structure way can collect a variety of screwdrivers into one, realizing the integration of a variety of screwdrivers and trigger body installation, which is convenient to use.

The structure of the force-applied rod is shown in Figure 3. Replaceable screwdriver heads of various types are installed inside the hollow force application lever, and the operator can take out the screwdriver head on its open side for use.

Both ends of the lever are knurled to provide aesthetic and antislip effects.

\section{(1))}

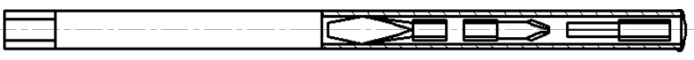

Figure 3. Schematic diagram of the structure of the forceapplying rod.

\subsubsection{Itinerary design.}

In order for this improved design product to move within a certain stroke under the action of spring force and external force applied by the operator, and eventually achieve the protection function of automatic ejection, parts are needed to limit its stroke.

As can be seen in Figure 4 of the overall structure, a sleeve part is utilized. The sleeve is connected to the chuck body by two symmetrical $180^{\circ}$ key slotted holes in the wall of 
the sleeve, and the lever is passed through the holes, because the lever is fixed, so that the chuck body can only move within the range of its own key slotted holes. The length of the through-hole is the maximum travel of the wrench when it works, and its value is designed according to the overall structure and working condition of the wrench and the specific situation.

The stroke is not strictly required in this improved design, as long as it meets the working requirements of the whole designed product. The specific working stroke integrates the size of the chuck wrench head, the size of the tool holder sleeve and the actual situation at work, and the design stroke $\mathrm{L}=35 \mathrm{~mm}$.

When designing the length of the stroke hole, it is necessary to take into account that the force rod passes through the stroke hole, because the diameter of the designed force rod is $11 \mathrm{~mm}$, so the length of the stroke hole is designed as the actual maximum working stroke plus the diameter of the force rod, which is $46 \mathrm{~mm}$.
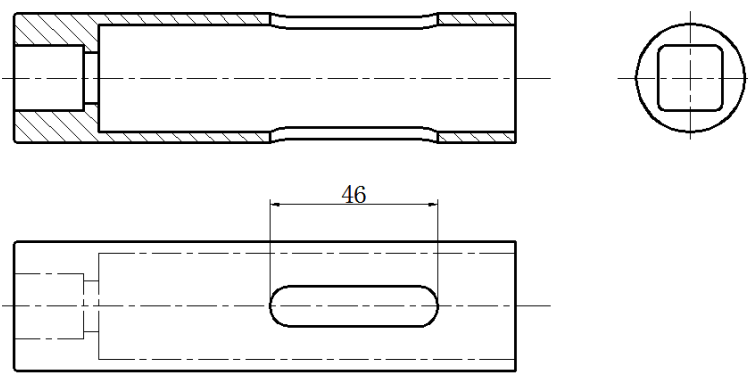

Figure 4. Tool holder wrench sleeve parts diagram.

\subsubsection{Spring design.}

The design of the spring is a key point of the new multiuse wrench. First of all, according to the work situation and specific conditions selected materials, the design, the use of the spring environment is room temperature environment, neither corrosive media nor bear a large impact load, so the use of general high-quality carbon steel can be selected after the material to determine the allowable stress. Spring material selection of 65 highquality carbon steel, heat treatment process for oil quenching, $400^{\circ} \mathrm{C} \sim 420^{\circ} \mathrm{C}$ tempering, hardness HRC42 HRC48 can be. Because the spring steel wire diameter is less than $8 \sim 10 \mathrm{~mm}$, so the use of cold roll method of manufacture, the spring ends and tight, grinding flat.

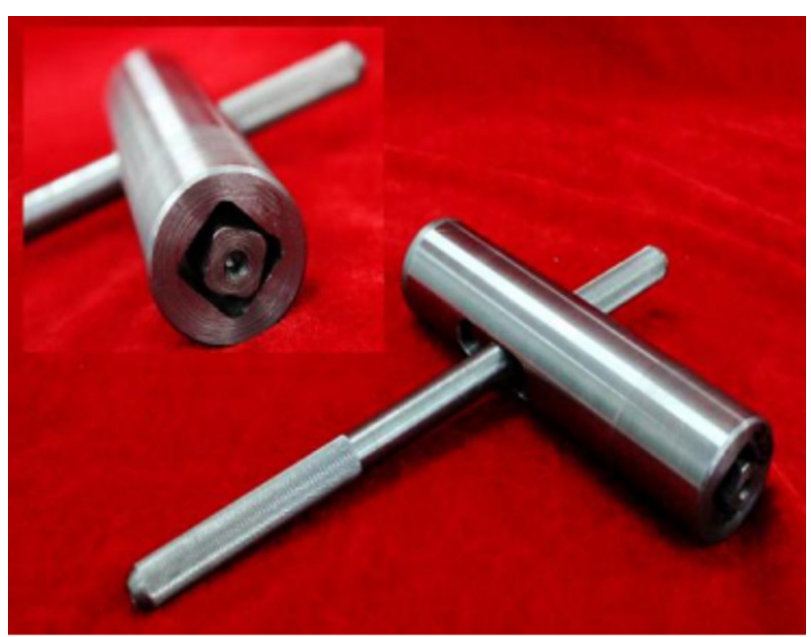

Figure 5. Physical drawing of the new dual-use wrench for lathe.

\section{Conclusion}

The new lathe multi-purpose safety wrench adopts reasonable structural design and improves the existing two types of wrenches, integrating the functions of chuck wrenches, tool holder wrenches and a variety of screwdrivers, and its volume is only the size of a wrench, with compact structure and convenient operation, so that the wrench will not be taken by mistake, saving the time of frequent wrench replacement, reducing labor intensity and improving production efficiency. More importantly, it avoids the safety hazards caused by forgetting to take off the wrench. It has a very significant effect on the longterm use of the equipment and the personal safety of the operator, etc. And the production cost is not high, which has a very great promotion value.

\section{Acknowledgments}

This work was financially supported by Shenyang Aerospace University Student's Platform for Innovation and Entrepreneurship Training Program fund. Project fund number: X202010143108.

First author: Peng Wang (2000.03--), male, Junior, the main research direction is aircraft manufacture engineering.

Corresponding author: Zhou Yan (1982.12--), male, engineer, main research direction is the application of numerical control technology and mechanical design research.

\section{References}

1. T.J. Luo, W.X. Pei, Design and application of safety chuck wrench, J. Machine Tools and Hydraulics, 2012,40(2):22-24

2. D.C. Gao,Application of safety devices in the improved design of lathe chuck wrenches, J. Heilongjiang science and technology information, 2015:44 
3. X. Zhang, W.L. Pan,Design and application of safety protection device for lathe chuck wrench, J. Silicon Valley, 2013(7):181

4. H. Wang, J.G. Zhang, The development of lathe chuck locking safety wrench, J. Small and medium-sized enterprise management and technology, 2010:310

5. T.Zhang, L.Wu, Multifunctional lathe safety wrench, J. Science 24 Hours, 2007:35-36

6. J.H. Zhang, Improved design of chuck wrench, J. Equipment management and maintenance, 2011:3536

7. Y.F. Zhou, Fundamentals of mechanical design. Beijing: Machinery Industry Press, 2008,pp. 84-332.

8. J.S. Wang, Mechanical general parts manual. Beijing: China Electric Power Publishing House, 2008,pp. 262-682. 